\title{
Knowledge, attitudes, practices and behaviors associated with female condoms in developing countries: a scoping review
}

This article was published in the following Dove Press journal:

Open Access Journal of Contraception

21 September 2015

Number of times this article has been viewed

\section{Lizzie Moore' \\ Mags Beksinska ${ }^{1,2}$ \\ Alnecia Rumphs ${ }^{3}$ \\ Mario Festin ${ }^{4}$ \\ Erica L Gollub ${ }^{3}$}

'MatCH Research (Maternal, Adolescent and Child Health Research), Department of Obstetrics and Gynaecology, University of the Witwatersrand, Westville, Durban, South Africa; ${ }^{2}$ Faculty of Epidemiology and Population Health, London School of Hygiene and Tropical Medicine, London, UK; ${ }^{3}$ Florida International University, Department of Epidemiology, Stempel College of Public Health and Social Work, Miami, FL, USA; ${ }^{4}$ World Health Organization, Special Program of Research, Development and Research Training in Human Reproduction, Department of Reproductive Health and Research, World Health Organization, Geneva, Switzerland

Correspondence: Erica L Gollub Florida International University, Department of Epidemiology, Stempel College of Public Health and Social Work, II 200 SW 8th Street, AHC 5-Rm 482, Miami, FL 33199, USA Email elgollub@gmail.com

\begin{abstract}
Women in developing countries are at high risk of HIV, sexually transmitted infections, and unplanned pregnancy. The female condom (FC) is an effective dual protective method regarded as a tool for woman's empowerment, yet supply and uptake are limited. Numerous individual, socioeconomic, and cultural factors influence uptake of new contraceptive methods. We reviewed studies of FC knowledge, attitudes, practices, and behaviors across developing countries, as well as available country-level survey data, in order to identify overarching trends and themes. High acceptability was documented in studies conducted in diverse settings among male and female FC users, with FCs frequently compared favorably to male condoms. Furthermore, FC introduction has been shown to increase the proportion of "protected" sex acts in study populations, by offering couples additional choice. However, available national survey data showed low uptake with no strong association with method awareness, as well as inconsistent patterns of use between countries. We identified a large number of method attributes and contextual factors influencing FC use/nonuse, most of which were perceived both positively and negatively by different groups and between settings. Male partner objection was the most pervasive factor preventing initial and continued use. Importantly, most problems could be overcome with practice and adequate support. These findings demonstrate the importance of accounting for contextual factors impacting demand in FC programming at a local level. Ongoing access to counseling for initial FC users and adopters is likely to play a critical role in successful introduction.
\end{abstract}

Keywords: condoms, HIV prevention, contraception, female condom, developing countries, behavior

\section{Introduction}

Women carry a disproportionate burden of $\mathrm{HIV}^{1}$ resulting from numerous physiological, socioeconomic, cultural, and political factors, including unbalanced gender norms impacting sexual negotiation..$^{2-7}$ Furthermore, over one-third of pregnancies in developing countries is unplanned, ${ }^{8}$ making unmet need for contraception a priority policy area. ${ }^{9}$

The female condom (FC) is the only available woman-initiated method for preventing HIV/sexually transmitted infections (STIs) and unintended pregnancy. ${ }^{10} \mathrm{It}$ has comparable dual protective efficacy to male condoms (MCs) ${ }^{11}$ and is frequently cited as a tool for women's empowerment. ${ }^{12-14}$ There are several FC models including FC2, The Woman's Condom, The Phoenurse, Cupid, Panty (Condon Femenino), Velvet, and VA w.o.w (Condom Feminine); all have common components with unique design features. ${ }^{15}$ Although the Female Health Company's FC2 (Chicago, IL, USA) 
is the only current model approved by the United States Food and Drug Administration (USFDA), Cupid has been prequalified for distribution by United Nations (UN) agen$\operatorname{cies}^{16}$ and others have commenced or are planning UN and USFDA applications. ${ }^{15}$

Over 20 years since its first USFDA approval, FC supply and uptake remains inadequate. ${ }^{17}$ Few established national programs exist, ${ }^{18-20}$ with low distribution ${ }^{21}$ often attributed to a lack of policy and donor support ${ }^{11,17,22}$ and relatively high procurement costs compared to $\mathrm{MCs},{ }^{11,23}$ despite long-term cost effectiveness. ${ }^{14,24,25}$ As FC options increase, the method may become more affordable, encouraging greater distribution and use. ${ }^{15}$

Increasing availability of new contraceptive methods does not automatically broaden choice. Uptake depends on the perceptions and experiences of potential users and the socioeconomic context, ${ }^{23,26}$ with culture and gender relations often having greater impact on acceptability than actual attributes of the method. ${ }^{13,27}$ Health system capacity and acceptance of new methods by service providers additionally influence uptake and sustained use. ${ }^{26,28}$ Any FC program evaluation must therefore consider acceptability in context, by exploring knowledge, attitudes, practices, and behaviors (KAPB) in a wide range of stakeholders. We conducted a scoping review ${ }^{29}$ of FC KAPB across developing countries to identify overarching themes linking contextual variables with these outcomes.

\section{Methods}

The format of our review was a scoping study. The purpose of a scoping review is to map a wide range of literature and to identify the nature, range, and extent of the evidence..$^{29,30}$ Scoping reviews differ from systematic reviews in their broad approach to a topic, purposive sampling frame, and identification of gaps in the literature. We searched MEDLINE without date restrictions for material available through January 2015, using the terms "female condom" or "female-initiated", to identify KAPB studies for male and female users and nonusers. We also searched for studies exploring perspectives of other stakeholders such as health care providers, although this information falls beyond the scope of this article. Countries in which research was identified are listed in Table 1. Titles or abstracts (where a decision could not be made on title alone) were screened for studies that discussed FC KAPB in developing nations. We included English-, French-, and Spanish-language articles. We also searched the Websites of major international organizations involved in FC programming and the survey database of the
Reproductive Health Supplies Coalition ${ }^{31}$ using the same inclusion criteria. We reviewed a compiled list of research studies at the Female Health Company Website (http://www. femalecondom.org/). ${ }^{32}$ All reference lists were reviewed; where potentially relevant additional material was unavailable online, we contacted authors or publishers to obtain a copy where possible. All sources were organized by country, and data were extracted onto standardized data abstraction forms that stratified KAPB variables by distinct user subpopulations. Data were then examined across countries to identify emerging themes and trends. The purpose of a scoping review is to map a wide range of literature and to identify the nature and extent of the evidence; ${ }^{29}$ thus, for each country, research articles for which there were available data on potential or actual users or providers were then selected for inclusion in this review.

\section{Results}

We identified 56 countries with national survey data (usually as Demographic and Health Surveys [DHS] $)^{33-41}$ and 34 countries with other types of material (peer-reviewed articles, governmental or nongovernmental organizational reports, or other gray literature). The frequency of articles and the depth of information varied considerably across countries. Table 1 shows the type of participants included in the FC studies, by country. Most research focused on women, particularly female sex workers (FSWs). Notably, only three studies $^{42-44}$ included men who have sex with men (MSM) (none addressed FC use by women for anal sex); we therefore include these data alongside those for female users, while perspectives of heterosexual male partners are considered separately. Sample sizes for quantitative reports ranged to 2,700 , although most contained a few hundred participants. Qualitative reports tended to be smaller, though most sample sizes exceeded 100. Table 2 "maps" the number and variety of peer-reviewed research compared with coverage by national survey data, organized by country and continent. Most countries listed provided DHS or other country-level survey data, but peer-reviewed data were considerably less frequent. The majority of peer-reviewed research emanated from Africa, where both qualitative and quantitative studies were available for several countries. By contrast, countries in the Americas were less well represented by quantitative studies. Single countries having the greatest number of peer-reviewed articles were South Africa (18 articles), the People's Republic of China (nine articles), and Zimbabwe (eight articles) (data not shown). Although most studies were conducted with the discontinued FC1, several demonstrated comparable 
Table I Availability of female condom research by participant type and country

\begin{tabular}{|c|c|c|c|c|c|}
\hline Country & Women/ couples & Men & FSWs & Providers & Other \\
\hline Bangladesh & - & & - & & \\
\hline Botswana & & & & - & \\
\hline Brazil & - & - & - & & 0 \\
\hline Burundi & - & & & & \\
\hline Cambodia & & & - & & \\
\hline Cameroon & & & - & & \\
\hline Central African Republic & - & - & & & \\
\hline People's Republic of China & - & - & - & - & 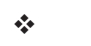 \\
\hline Dominican Republic & & - & - & & \\
\hline El Salvador & & & - & & \\
\hline Ghana & - & & - & - & $\star$ \\
\hline India & - & - & - & - & $\star$ \\
\hline \multicolumn{6}{|l|}{ Indonesia } \\
\hline Ivory Coast & घ & & - & & \\
\hline Kenya & - & & - & - & $\star$ \\
\hline Madagascar & & & - & & \\
\hline Malawi & - & - & - & & \\
\hline Mexico & - & - & - & & \\
\hline Mozambique & & & - & & 0 \\
\hline Namibia & - & - & & & 0 \\
\hline Nicaragua & & & - & & \\
\hline Nigeria & घ & - & - & - & \\
\hline Papua New Guinea & & & - & & \\
\hline Rwanda & घ & - & & & \\
\hline South Africa & - & - & & - & $\star$ \\
\hline Swaziland & & & - & & \\
\hline Tanzania & घ & & - & & \\
\hline Thailand & - & - & - & & \\
\hline Tunisia & & & - & & \\
\hline Turkey & घ & & & & 0 \\
\hline Uganda & - & - & घ & - & \\
\hline Vietnam & - & & - & & \\
\hline Zambia & - & - & & & $0, \odot$ \\
\hline Zimbabwe & घ & घ & ! & - & \\
\hline Total & 22 & 15 & 25 & 9 & 10 \\
\hline
\end{tabular}

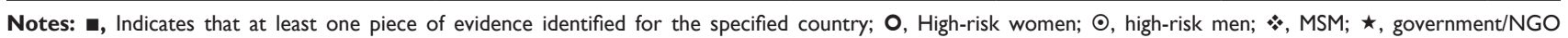
stakeholders.

Abbreviations: FSWs, female sex workers; n, number; MSM, men who have sex with men; NGO, nongovernmental organization.

acceptability between various brands. ${ }^{45-50}$ Reports on provider attitudes were limited; our search turned up only six peer-reviewed articles. We made reference to these findings where relevant and integrated with data from users.

\section{Knowledge and awareness}

National data on FC knowledge, primarily from DHS, were available for 56 countries. These demonstrated widely varying between-country awareness (Table 3 ). The prevalence of FC awareness within countries was consistently lower than that of MCs; for example in the Democratic Republic of the Congo, $43 \%$ and $82 \%$ of women had knowledge of FCs and MCs, respectively. ${ }^{51}$ The proportion of women across all countries having heard of FC (FC knowledge) was also generally lower (47\%) than for the oral contraceptive (OC) $(85 \%)$, injectables $(80 \%)$, and the intrauterine device (IUD) (55\%) (data not shown). ${ }^{33}$ In general, men demonstrated slightly higher FC awareness (54\%) than women (47\%), but they exhibited lower levels of knowledge on other methods, as compared with that of women: OC (76\%); injectables (67\%); or the IUD (38\%) (data not shown). ${ }^{33}$ FC knowledge also varied within countries (where available), ${ }^{52}$ for example, in India, 13\% of urban women reported awareness compared to $6 \%$ of rural women. ${ }^{41}$ Some studies supported the contention that greater awareness of and counseling on FC would increase interest and possible use ${ }^{53,54}$ nevertheless, it is clear that $\mathrm{FC}$ awareness alone appears to be insufficient to stimulate uptake. ${ }^{55}$ For example $91.4 \%$ of women interviewed in the 
Table 2 Comparison of national survey data and peer-reviewed research by type, country, and continent

\begin{tabular}{|c|c|c|c|c|c|c|}
\hline Continent & Country & Quantitative $^{a}$ & Qualitative $^{a}$ & Mixed $^{\mathrm{a}}$ & Any peer review & National survey \\
\hline \multirow[t]{38}{*}{ Africa } & Benin & & & & & - \\
\hline & Botswana & & ! & & घ & \\
\hline & Burkina Faso & & & & & ! \\
\hline & Burundi & & & & & घ \\
\hline & Cameroon & - & & & घ & - \\
\hline & CAR & - & & & - & \\
\hline & Chad & & & & & - \\
\hline & Comoros & & & & & - \\
\hline & Congo & & & & & घ \\
\hline & DRC & & & & & घ \\
\hline & Eritrea & & & & & - \\
\hline & Ethiopia & & & & & - \\
\hline & Gabon & & & & & - \\
\hline & Ghana & & & & & - \\
\hline & Guinea & & & & & - \\
\hline & Ivory Coast & & - & & ! & - \\
\hline & Kenya & घ & 匹 & & - & - \\
\hline & Lesotho & & & & & - \\
\hline & Liberia & & & & & - \\
\hline & Madagascar & 匹 & & & - & - \\
\hline & Malawi & - & & & - & - \\
\hline & Mali & & & & & - \\
\hline & Mauritania & & & & & - \\
\hline & Mozambique & & & & & - \\
\hline & Namibia & & - & & - & - \\
\hline & Niger & & & & & - \\
\hline & Nigeria & 匹 & घ & - & घ & - \\
\hline & Rwanda & - & & & - & - \\
\hline & Senegal & & & & & ! \\
\hline & Sierra Leone & & & & & - \\
\hline & South Africa & 匹 & घ & - & - & - \\
\hline & STP & & & & & ! \\
\hline & Swaziland & & घ & & a & - \\
\hline & Tanzania & घ & & & - & - \\
\hline & Tunisia & घ & & & - & \\
\hline & Uganda & - & - & & - & ! \\
\hline & Zambia & घ & . & & - & ! \\
\hline & Zimbabwe & - & - & - & - & - \\
\hline \multirow[t]{11}{*}{ Americas } & Brazil & - & & - & - & \\
\hline & Dominican Republic & & a & & घ & ! \\
\hline & El Salvador & & 匹 & & घ & - \\
\hline & Guatemala & & & & & - \\
\hline & Guyana & & & & & - \\
\hline & Haiti & & & & & ! \\
\hline & Honduras & & & & & ! \\
\hline & Mexico & 匹 & 匹 & - & - & \\
\hline & Nicaragua & & - & & - & ! \\
\hline & Paraguay & & & & & ! \\
\hline & Peru & & & & & - \\
\hline \multirow[t]{8}{*}{ Asia } & Bangladesh & घ & & & घ & \\
\hline & Cambodia & & & - & घ & ! \\
\hline & China & - & & ! & - & \\
\hline & India & - & & & - & - \\
\hline & Jordan & & & & & - \\
\hline & Kazakhstan & & & & & ! \\
\hline & Kyrgyz Republic & & & & & ! \\
\hline & Philippines & & & & & घ \\
\hline
\end{tabular}


Table 2 (Continued)

\begin{tabular}{|c|c|c|c|c|c|c|}
\hline Continent & Country & Quantitative $^{\mathrm{a}}$ & Qualitative $^{\mathrm{a}}$ & Mixed $^{a}$ & Any peer review & National survey \\
\hline & Tajikistan & & & & & घ \\
\hline & Thailand & a & & a & a & \\
\hline & Timor-Leste & & & & & $\square$ \\
\hline & Turkmenistan & & & & & $\mathbf{\square}$ \\
\hline Australia & PNG & & & & & घ \\
\hline \multirow[t]{2}{*}{ Europe } & Albania & & & & & घ \\
\hline & Turkey & घ & & & घ & घ \\
\hline
\end{tabular}

Note: ancludes only peer-reviewed research. $\mathbf{a}$ Indicates that at least one piece of evidence of this type identified for the specified country.

Abbreviations: CAR, Central African Republic; DRC, Democratic Republic of the Congo; STP, Sao Tome and Principe; PNG, Papua New Guinea.

most recent Swaziland DHS had heard of FCs, yet only $46 \%$ knew where to source one. ${ }^{56}$

\section{General perceptions and attitudes}

High acceptability was documented across numerous and varied settings where studies were undertaken among women, men, and couples who tried the device. ${ }^{45,57-59}$ For example, studies introducing FCs in South Africa and Kenya reported that over $85 \%$ of male and female participants expressed an intention to use FCs in the future, and even more would recommend them to friends. ${ }^{60,61} \mathrm{~A}$ large number of studies among FSWs suggest that FCs are consistently acceptable to this population, ${ }^{62,63}$ for example, in Papua New Guinea, $90 \%$ of female participants engaging in transactional sex reported "liking" the FC. ${ }^{64}$ In India, 83\% of MSM FC users said they would continue to use the device. ${ }^{42}$

Numerous studies reported that FCs compared favorably with MCs. For example, $80 \%$ of women in a South African short-term crossover trial favored the $\mathrm{FC} 1$ and $\mathrm{FC} 2$ over $\mathrm{MCs} ;{ }^{50}$ in a Nigerian study, many more participants accepted and paid for FCs than MCs ( $8 \%$ versus 1\%, respectively) following a provider training intervention. ${ }^{65}$ Women and MSM also gauged FC acceptability against the MC, based on previous negative experiences or perceived superior safety, strength, or effectiveness. ${ }^{42,43,66}$ Advantages over the MC were frequently highlighted, such as comfort, lack of male responsibility, enhanced sexual pleasure, and potential use during menstruation. ${ }^{67-71}$ Contraceptive properties were highlighted in El Salvador, Swaziland, and Zimbabwe. ${ }^{68-70}$

Several studies did not distinguish between factors influencing initial uptake or continued use, but they reported overall responses. The same method attributes of FCs were perceived both positively and negatively by different groups and between settings (Table 4). Similarly, environmental/ contextual factors both positively and negatively influenced uptake in different settings (Table 5). Although few studies commented on patterns of acceptability, several contradictory trends existed between countries based on marital status, education, and occupation, ${ }^{55,58,72-76}$ thus supporting the conclusion of a 2006 systematic review that predictors of acceptability are not generalizable across cultural contexts. ${ }^{77}$

Some studies reported that stigmatized notions of the FC impeded initial use and were widely expressed by men, women, and health care providers, ${ }^{52,54,73,78,79}$ linking the device with infidelity and commercial sex work, even when marketed as a contraceptive for stable couples. ${ }^{54,61,66,68,78-81}$ For example, a quarter of female participants in a Kenyan study felt that using or carrying an $\mathrm{FC}$ was synonymous with unfaithfulness. ${ }^{61}$ Researchers in Zimbabwe commented that "just like the male condom, the $[\mathrm{FC}]$ was seen as a threat to intimacy and commitment, in that requesting them would introduce an element of distrust and suspicion of infidelity and promiscuity" ${ }^{82}$

Cost was a frequently cited acceptability barrier. Although both clients and providers often indicated that the FC should be freely available, ${ }^{83-86}$ most conceded that they would be willing to pay a highly subsidized price, ${ }^{67,87-90}$ usually equal to that of MCs. ${ }^{66,91,92}$ FSWs in Malawi noted that free FCs were only available in hospitals. ${ }^{84}$ By contrast, in Cameroon, where intensive $\mathrm{FC}$ availability campaigns and a massive decrease in price has recently occurred, a relatively high everuse rate among female high school students (8\%) suggests that price significantly influences acceptability. ${ }^{93}$

Lack of availability and access were the most frequently cited contributing factors to FC non-use, discontinuation and reuse, across diverse subpopulations, including students, FSWs and women in the general population. For example, $43 \%$ of Rwandan undergraduates agreed that they would use FC if available, ${ }^{54}$ and only one-quarter of Chinese family planning clients thought that $\mathrm{FC}$ was easy to obtain. ${ }^{95}$ Furthermore, lack of access or availability were frequently cited by healthcare providers as a reason not to counsel clients on FC use. $22 \%$ of Zimbabwean health care workers indicated they would offer FCs more frequently if availability was improved (note that the terms availability and access were used variously by different 
Table 3 Prevalence of female condom awareness and use ${ }^{a}$ by country (listed by prevalence of awareness) from national survey data

\begin{tabular}{|c|c|c|c|c|c|}
\hline \multirow[t]{2}{*}{ Country } & \multirow{2}{*}{$\begin{array}{l}\text { Survey } \\
\text { date }\end{array}$} & \multicolumn{2}{|c|}{ Awareness $^{c}$} & \multirow{2}{*}{$\begin{array}{l}\text { Ever } \\
\text { use }\end{array}$} & \multirow{2}{*}{$\begin{array}{l}\text { Current } \\
\text { use }\end{array}$} \\
\hline & & Women & Men & & \\
\hline \multirow[t]{2}{*}{ Namibia } & 2013 & $94.2 \%$ & $92.5 \%$ & & $0.5 \%$ \\
\hline & 2006 & $83.0 \%$ & $82.3 \%$ & $6.4 \%$ & $0.3 \%$ \\
\hline \multirow[t]{3}{*}{ Swaziland } & 2006 & $91.3 \%$ & $84.1 \%$ & $3.3 \%$ & $0.1 \%$ \\
\hline & 2010 & & & & $0.4 \%$ \\
\hline & MICS & & & & \\
\hline Lesotho & 2009 & $86.6 \%$ & $77.9 \%$ & & $0.2 \%$ \\
\hline Malawi & 2010 & $86.0 \%$ & $84.9 \%$ & $1.2 \%$ & $0.1 \%$ \\
\hline Zimbabwe & 2010 & $83.9 \%$ & $87.4 \%$ & & $0.3 \%$ \\
\hline Rwanda & 2010 & $82.4 \%$ & $79.9 \%$ & & \\
\hline Gabon & 2012 & $81.7 \%$ & $78.2 \%$ & & $0.1 \%$ \\
\hline Haiti & 2012 & $81.5 \%$ & & & $<0.1 \%$ \\
\hline Ghana & 2008 & $81.3 \%$ & $86.3 \%$ & $0.7 \%$ & $<0.1 \%$ \\
\hline Guyana & 2009 & $78.4 \%$ & $69.9 \%$ & $1.5 \%$ & $<0.1 \%$ \\
\hline \multirow[t]{3}{*}{ South Africa } & 2008 & $77.8 \%^{\mathrm{d}}$ & $72.1 \%$ & $7.2 \%^{d}$ & \\
\hline & NHPIBCS & & & & \\
\hline & 2003 & $53.2 \%$ & $56.4 \%$ & $2.6 \%$ & $0.2 \%$ \\
\hline Dominican & 2013 & $74.8 \%$ & & & \\
\hline Republic & 2007 & $52.2 \%$ & & $0.6 \%$ & \\
\hline Tanzania & 2010 & $72.5 \%$ & $73.4 \%$ & & $<0.1 \%$ \\
\hline Uganda & 2011 & $70.5 \%$ & $81.4 \%$ & & \\
\hline Cameroon & 2011 & $70.4 \%$ & $77.0 \%$ & & $0.1 \%$ \\
\hline Sierra Leone & 2013 & $69.5 \%$ & $64.7 \%$ & & $<0.1 \%$ \\
\hline Liberia & 2013 & $69.3 \%$ & $56.1 \%$ & & \\
\hline Burundi & 2010 & $69.1 \%$ & $66.4 \%$ & & $<0.1 \%$ \\
\hline Congo & 2011 & $68.3 \%$ & $85.0 \%$ & & $<0.1 \%$ \\
\hline Zambia & 2007 & $65.8 \%$ & $65.5 \%$ & $1.2 \%$ & $<0.1 \%$ \\
\hline Sao Tome and & 2008 & $58.6 \%$ & $61.1 \%$ & $0.5 \%$ & \\
\hline \multicolumn{6}{|l|}{ Principe } \\
\hline Kenya & 2008 & $57.6 \%$ & $61.5 \%$ & $0.6 \%$ & $<0.1 \%$ \\
\hline Comoros & 2012 & $54.8 \%$ & $60.8 \%$ & & $<0.1 \%$ \\
\hline Ivory Coast & 2012 & $54.4 \%$ & $63.0 \%$ & & $<0.1 \%$ \\
\hline Paraguay & 2004 & $54.0 \%$ & & & \\
\hline Burkina Faso & 2010 & $47.8 \%$ & $52.5 \%$ & & $<0.1 \%$ \\
\hline Mozambique & 2011 & $45.3 \%$ & $77.1 \%$ & & $0.1 \%$ \\
\hline Peru & 2011 & $44.7 \%$ & & $0.2 \%$ & \\
\hline Honduras & 2011 & $44.6 \%$ & $48.8 \%$ & $0.4 \%$ & $<0.1 \%$ \\
\hline Democratic & 2013 & $43.2 \%$ & $52.8 \%$ & & $0.1 \%$ \\
\hline \multicolumn{6}{|l|}{ Republic of the } \\
\hline \multicolumn{6}{|l|}{ Congo } \\
\hline Papua New Guinea & 2006 & $40.1 \%$ & $46.1 \%$ & $0.6 \%$ & \\
\hline Senegal & 2010 & $37.2 \%$ & $44.0 \%$ & & $<0.1 \%$ \\
\hline El Salvador & 2008 & $36.8 \%$ & & $0.3 \%$ & \\
\hline Mali & 2012 & $35.7 \%$ & $38.7 \%$ & & $<0.1 \%$ \\
\hline Benin & 2012 & $34.9 \%$ & $47.8 \%$ & & $<0.1 \%$ \\
\hline Nicaragua & 2001 & $32.9 \%$ & & $0.3 \%$ & \\
\hline Ethiopia & 2011 & $31.9 \%$ & $39.1 \%$ & & $<0.1 \%$ \\
\hline \multirow[t]{2}{*}{ Nigeria } & 2013 & $28.6 \%$ & $32.8 \%$ & & $<0.1 \%$ \\
\hline & 2008 & $14.7 \%$ & $25.9 \%$ & $0.2 \%$ & $<0.1 \%$ \\
\hline Guinea & 2012 & $27.5 \%$ & & & $<0.1 \%$ \\
\hline Guatemala & 2002 & $25.0 \%$ & & & \\
\hline Cambodia & 2010 & $23.5 \%$ & & & $<0.1 \%$ \\
\hline Eritrea & 2002 & $23.0 \%$ & & $0.1 \%$ & $<0.1 \%$ \\
\hline Philippines & 2013 & $20.0 \%$ & & & \\
\hline
\end{tabular}

(Continued)
Table 3 (Continued)

\begin{tabular}{|c|c|c|c|c|c|}
\hline \multirow[t]{2}{*}{ Country } & \multirow{2}{*}{$\begin{array}{l}\text { Survey } \\
\text { date }\end{array}$} & \multicolumn{2}{|c|}{ Awareness $^{c}$} & \multirow{2}{*}{$\begin{array}{l}\text { Ever } \\
\text { use }\end{array}$} & \multirow{2}{*}{$\begin{array}{l}\text { Current } \\
\text { use }\end{array}$} \\
\hline & & Women & Men & & \\
\hline Kyrgyz Republic & 2012 & $19.5 \%$ & $21.5 \%$ & & \\
\hline Jordan & 2012 & $18.7 \%$ & & & \\
\hline Madagascar & 2008 & $18.5 \%$ & $21.2 \%$ & $0.1 \%$ & $<0.1 \%$ \\
\hline Kazakhstan & 1999 & $17.9 \%$ & $4.9 \%$ & $0.1 \%$ & \\
\hline Albania & 2008 & $15.2 \%$ & $8.9 \%$ & $0.3 \%$ & $<0.1 \%$ \\
\hline Niger & 2012 & $15.2 \%$ & $17.8 \%$ & & $<0.1 \%$ \\
\hline \multirow[t]{2}{*}{ Turkey } & 2003 & $13.5 \%$ & & & \\
\hline & & $13.4 \%^{\mathrm{f}}$ & & & \\
\hline Timor-Leste & 2009 & $10.4 \%$ & $10.4 \%$ & $<0.1 \%$ & \\
\hline \multirow[t]{2}{*}{ India } & 2006 & $8.3 \%$ & $16.8 \%$ & $<0.1 \%$ & $<0.1 \%$ \\
\hline & NFHS & & & & \\
\hline Tajikistan & 2012 & $7.2 \%$ & & & \\
\hline Chad & 2004 & $7.1 \%$ & $27.3 \%$ & $<0.1 \%$ & $<0.1 \%$ \\
\hline Turkmenistan & 2000 & $6.3 \%$ & & $<0.1 \%$ & \\
\hline Mauritania & 2000 & $5.3 \%$ & $7.5 \%$ & $<0.1 \%$ & \\
\hline
\end{tabular}

Notes: aRefers to all women 15-49 years of age, unless otherwise indicated; brefers to Demographic and Health Survey, unless otherwise indicated; cawareness of female condoms as a contraceptive method (percentage of all respondents, currently married respondents, and sexually active unmarried respondents ages 15-49 years who know of any contraceptive method, by specific method); 'sexually active women over 15 years of age; 'ever-married women; fcurrently married women.

Abbreviations: MICS, Multiple Indicator Cluster Survey; NHPIBCS, National HIV Prevalence, Incidence, Behavior and Communication Survey; NFHS, National Family Health Survey.

authors with a great degree of overlap in meaning; we have adhered to the original study terms where possible). ${ }^{94}$

\section{Initial use}

FC acceptance in a trial setting varied. In a large Brazilian study, $90 \%$ of participants initially introduced to the device had used the FC at least once at the 90-day follow-up visit (hereafter, referred to as follow-up) ${ }^{58}$ whereas in a Kenyan study, less than half of the participants recruited through HIV counseling and testing centers were willing to use the FC. ${ }^{61}$ The most common reason for never-use was the fear of partner reaction or partner refusal. ${ }^{60,61,78,96}$ Appearance ${ }^{59,61,81,92}$ and lack of perceived need ${ }^{61}$ were also cited as initial barriers.

Triggers for initial FC use included novelty, ${ }^{43,61,67,71,96}$ dual protective properties, partners' or clients' unwillingness to use MCs, ${ }^{42,67,92}$ and the feeling that it was woman-initiated. ${ }^{43,61,67,97}$ Promotion and support were frequently-reported facilitators of initial use. ${ }^{22,53,71,81,88,98-100}$ For example, counselor training and peer support groups in Kenya helped women introduce the FC into sexual relationships..$^{98,101}$ In Zimbabwe, having observed an FC demonstration in a hair salon more than doubled a woman's likelihood of having tried the device. ${ }^{22} \mathrm{In}$ Tanzania, a mass media campaign, which triggered communication about the FC between partners, increased women's intention to use the device. ${ }^{99}$ 
Table 4 Method attributes (actual and perceived) of FCs influencing acceptability, uptake and/or continued use

\begin{tabular}{|c|c|c|}
\hline Attribute & $\begin{array}{l}\text { Positive perceptions/facilitators of use } \\
\text { (countries where relevant evidence was identified) }\end{array}$ & $\begin{array}{l}\text { Negative perceptions/barriers to use } \\
\text { (countries where relevant evidence was identified) }\end{array}$ \\
\hline Appearance & $\begin{array}{l}\text { - General appearance } \\
\text { (People's Republic of China) } \\
\text { - Large size } \\
\text { (South Africa, Uganda, El Salvador, Nicaragua) } \\
\text { - Smell (preferred to MC) } \\
\text { (Burundi, El Salvador, Nicaragua) } \\
\text { - Natural feel } \\
\text { (El Salvador, Nicaragua, Nigeria) }\end{array}$ & $\begin{array}{l}\text { - Generally unattractive size and shape } \\
\text { (Brazil, Ghana, India, Kenya, South Africa, Uganda, Zimbabwe, } \\
\text { El Salvador, Cambodia, People's Republic of China, Dominican } \\
\text { Republic, Thailand, Vietnam, Nigeria, Nicaragua) } \\
\text { - Too thick } \\
\text { (Ghana) } \\
\text { - Too big/loose/long } \\
\text { (India, Kenya, South Africa, Uganda, Bangladesh, } \\
\text { Thailand, Nigeria) } \\
\text { - Rings are confusing } \\
\text { (South Africa) } \\
\text { - Smell } \\
\text { (South Africa) }\end{array}$ \\
\hline $\begin{array}{l}\text { Different than } \\
\text { other methods } \\
\text { Lubrication }\end{array}$ & $\begin{array}{l}\text { - Novelty factor } \\
\text { (India, Kenya, People's Republic of China, CAR, Nigeria) } \\
\text { - Well lubricated (better than MC) } \\
\text { (Brazil, Ghana, India, Uganda, Burundi, El Salvador, } \\
\text { Swaziland, Nicaragua) }\end{array}$ & $\begin{array}{l}\text { - More complicated than other contraceptives } \\
\text { (Vietnam, Zimbabwe, South Africa) } \\
\text { - Overlubricated } \\
\text { (Namibia, South Africa, Tanzania, Zimbabwe, People's } \\
\text { Republic of China, Dominican Republic, Thailand) } \\
\text { - Fear of adverse effects of lubricant } \\
\text { (Burundi) }\end{array}$ \\
\hline Insertion/use & $\begin{array}{l}\text { - Easy/comfortable to insert and use during sex/“natural feel” } \\
\text { (compared to MC) } \\
\text { (India, Kenya, Namibia, South Africa, } \\
\text { Zimbabwe, Burundi, El Salvador, Mexico, Cameroon, } \\
\text { Cambodia, Dominican Republic, Thailand, Vietnam, Nigeria) } \\
\text { - Allows sex in any position without technical difficulties } \\
\text { (Brazil) } \\
\text { - Permitting use when penis is not erect } \\
\text { (El Salvador, Nicaragua) }\end{array}$ & $\begin{array}{l}\text { - Difficult to insert/remove } \\
\text { (Brazil, Namibia, Nigeria, PNG, Zimbabwe, Burundi, } \\
\text { El Salvador, Bangladesh, People's Republic of China, } \\
\text { Swaziland, Thailand, Nicaragua, CAR, Tunisia) } \\
\text { - Takes too long to insert } \\
\text { (Vietnam) } \\
\text { - Need privacy to insert } \\
\text { (India, Swaziland) } \\
\text { - Noise (FCI only) } \\
\text { (Brazil, Ghana, Namibia, PNG, South Africa, Burundi, } \\
\text { Cambodia, Dominican Republic, CAR, Malawi) } \\
\text { - Messy } \\
\text { (Kenya) } \\
\text { - Technical difficulties during sex/method failure } \\
\text { (Brazil, Ghana, Nigeria, Zimbabwe, Burundi, Cambodia) } \\
\text { - Fear of potential technical difficulties } \\
\text { (Burundi, People's Republic of China, Nigeria, South Africa) } \\
\text { - Female or male partner discomfort } \\
\text { (Brazil, India, Nigeria, Zimbabwe, Burundi, El Salvador, } \\
\text { Bangladesh, People's Republic of China, Dominican Republic, } \\
\text { Swaziland, Thailand, South Africa) } \\
\text { - Concerns about potential discomfort } \\
\text { (South Africa, Tanzania, Burundi, El Salvador, Nicaragua) } \\
\text { - Problems/discomfort related to inner/outer ring } \\
\text { (Ghana, India, South Africa, Zimbabwe, Burundi, Cameroon, } \\
\text { Vietnam, El Salvador, Nicaragua, CAR) } \\
\text { - Itching sensations } \\
\text { (CAR) } \\
\text { Bleeding/burning/allergy } \\
\text { (El Salvador, Nicaragua) }\end{array}$ \\
\hline Timing of use & $\begin{array}{l}\text { - Ability to insert before sex (prior to drinking alcohol, prior } \\
\text { to man getting an erection) } \\
\text { (Brazil, Ghana, South Africa, Zimbabwe, El Salvador, } \\
\text { Cambodia, Swaziland, Nicaragua) }\end{array}$ & $\begin{array}{l}\text { Perception that FC must be inserted several hours } \\
\text { before sex } \\
\text { (South Africa) } \\
\text { - Insertion interrupts the sex act } \\
\text { (Zimbabwe) }\end{array}$ \\
\hline
\end{tabular}


Table 4 (Continued)

\begin{tabular}{|c|c|c|}
\hline Attribute & $\begin{array}{l}\text { Positive perceptions/facilitators of use } \\
\text { (countries where relevant evidence was identified) }\end{array}$ & $\begin{array}{l}\text { Negative perceptions/barriers to use } \\
\text { (countries where relevant evidence was identified) }\end{array}$ \\
\hline $\begin{array}{l}\text { Practice } \\
\text { improves } \\
\text { confidence }\end{array}$ & $\begin{array}{l}\text { - Comfort and ease of use improves with practice } \\
\text { (Brazil, Ghana, Namibia, South Africa, Zimbabwe, Cameroon, } \\
\text { Cambodia, El Salvador, Swaziland, Thailand, Vietnam) }\end{array}$ & $\begin{array}{l}\text { - Requires practice to use with confidence } \\
\text { (Cambodia) } \\
\text { - Discomfort persisted with repeated use } \\
\text { (South Africa) }\end{array}$ \\
\hline $\begin{array}{l}\text { Safety and } \\
\text { effectiveness }\end{array}$ & $\begin{array}{l}\text { - Perceived strength (compared to MC) } \\
\text { (Brazil, India, South Africa, Zimbabwe, El Salvador, Cambodia, } \\
\text { People's Republic of China, Dominican Republic, Swaziland, } \\
\text { Nicaragua) } \\
\text { - Perceived flexibility (compared to MC) } \\
\text { (South Africa) } \\
\text { - Perceived safety (compared to MC) } \\
\text { (Brazil, Zimbabwe, Thailand, CAR, South Africa) } \\
\text { - FC cannot get lost inside the body } \\
\text { (Cambodia) } \\
\text { - Perceived effectiveness in pregnancy/STI prevention } \\
\text { (compared to MC) } \\
\text { (India, South Africa, Uganda, Burundi, El Salvador, People's } \\
\text { Republic of China, Dominican Republic, Vietnam, Nicaragua) } \\
\text { - Perceived effectiveness in pregnancy/STI prevention } \\
\text { (Rwanda, South Africa, Nigeria) } \\
\text { - Reduced slippage } \\
\text { (El Salvador, Nicaragua) } \\
\text { - Better protection from STIs (covers the outer part of the } \\
\text { vagina and labia) } \\
\text { (El Salvador, Nicaragua) }\end{array}$ & $\begin{array}{l}\text { - Doubts about effectiveness (compared to MC) } \\
\text { (Kenya, Zimbabwe) } \\
\text { - Fear of losing FC in reproductive tract/abdomen } \\
\text { (Ghana, Zimbabwe, People's Republic of China, South Africa) } \\
\text { - Lack of trust in effectiveness against HIV/STI risk among } \\
\text { males } \\
\text { (Nigeria) } \\
\text { - Method failure resulting in pregnancy } \\
\text { (Nigeria) }\end{array}$ \\
\hline $\begin{array}{l}\text { Dual protective } \\
\text { properties }\end{array}$ & $\begin{array}{l}\text { - Provides dual protection } \\
\text { (Kenya, South Africa, Uganda, Zimbabwe, Burundi, People's } \\
\text { Republic of China, Dominican Republic, Swaziland, Nigeria) }\end{array}$ & \\
\hline Pleasure & $\begin{array}{l}\text { - Enhanced sexual pleasure for woman or man (including by } \\
\text { clitoral stimulation from the external ring), preferred over } \\
\text { MC for sexual pleasure } \\
\text { (Brazil, Ghana, India, Kenya, Zimbabwe, Burundi, } \\
\text { Swaziland, CAR) } \\
\text { - Does not affect sexual pleasure } \\
\text { (Kenya, South Africa, Vietnam, CAR) } \\
\text { - Couples can stay together for longer after ejaculation } \\
\text { (Kenya, South Africa) }\end{array}$ & $\begin{array}{l}\text { - Reduced sexual sensation/pleasure for woman or man } \\
\text { (Ghana, India, Kenya, Nigeria, South Africa, Burundi, People's } \\
\text { Republic of China, Dominican Republic) } \\
\text { - Perception that it would interfere with sexual pleasure } \\
\text { (Uganda) } \\
\text { - Outer ring makes genitalia inaccessible } \\
\text { (Uganda, Zimbabwe) }\end{array}$ \\
\hline $\begin{array}{l}\text { Woman } \\
\text { initiated }\end{array}$ & $\begin{array}{l}\text { - Increases woman's control and sexual agency } \\
\text { (Brazil, Ghana, India, Kenya) (Namibia, South Africa, Uganda, } \\
\text { Zimbabwe, Burundi, Mexico, Bangladesh, People's Republic of } \\
\text { China, Dominican Republic, Swaziland, Thailand, Vietnam, Nigeria) }\end{array}$ & \\
\hline Covert use & $\begin{array}{l}\text { Ability to use covertly } \\
\text { (Brazil, Uganda, Cambodia, Dominican Republic, Swaziland, } \\
\text { El Salvador, Nicaragua) }\end{array}$ & $\begin{array}{l}\text { - Inability to use covertly } \\
\text { (Uganda, Zimbabwe) }\end{array}$ \\
\hline Other & $\begin{array}{l}\text { - Ability to use during menstruation } \\
\text { (Brazil, El Salvador, Nicaragua) } \\
\text { - Offers an alternative to MC (for those who cannot/prefer not } \\
\text { to use MCs or when MC is not available at the time of sex } \\
\text { (Brazil, Ghana, Nigeria, Swaziland, El Salvador, Nicaragua, } \\
\text { Nigeria, South Africa) } \\
\text { - Offers alternative protection when nothing else is available } \\
\text { (Zimbabwe) } \\
\text { - Offer alternative to hormonal contraception } \\
\text { (Swaziland, El Salvador) } \\
\text { - Reuse possible } \\
\text { (Burundi, India) }\end{array}$ & $\begin{array}{l}\text { - Inconvenient/long-term use not feasible } \\
\text { (Burundi, People's Republic of China, Thailand) } \\
\text { - Not suitable for some traditional sexual practices } \\
\text { (Burundi) }\end{array}$ \\
\hline
\end{tabular}

Abbreviations: MC, male condom; CAR, Central African Republic; PNG, Papua New Guinea; FC, female condom; STI, sexually transmitted infection. 
Table 5 Contextual/environmental factors influencing FC acceptability, uptake, and/or continued use (excluding availability)

\begin{tabular}{|c|c|c|}
\hline Factor & $\begin{array}{l}\text { Facilitators } \\
\text { (countries where relevant evidence was identified) }\end{array}$ & $\begin{array}{l}\text { Barriers } \\
\text { (countries where relevant evidence was identified) }\end{array}$ \\
\hline $\begin{array}{l}\text { Experience with } \\
\text { condoms }\end{array}$ & $\begin{array}{l}\text { - Familiarity with MC use } \\
\text { (Zambia, Bangladesh) }\end{array}$ & $\begin{array}{l}\text { - Lack of knowledge/experience with condoms } \\
\text { (Kenya) } \\
\text { - Unfamiliarity with FC/lack of exposure } \\
\text { (South Africa, El Salvador, Swaziland) } \\
\text { - Preference for MC } \\
\text { (Nigeria, Zambia, Thailand, South Africa) } \\
\text { - Dislike of condoms in general } \\
\text { (Nigeria, Uganda) }\end{array}$ \\
\hline $\begin{array}{l}\text { Perceived } \\
\text { personal risk } \\
\text { Relationship or } \\
\text { gender dynamics }\end{array}$ & $\begin{array}{l}\text { - Perceived risk of STI/HIV infection } \\
\text { (Bangladesh, People's Republic of China, Nigeria, Zimbabwe, } \\
\text { CAR, Tunisia, South Africa) } \\
\text { - Ability to discuss FC with a partner } \\
\text { (Tanzania, Zambia) } \\
\text { - More comfortable using FC with (regular) paying client than } \\
\text { nonpaying partner } \\
\text { (Brazil, Ghana, El Salvador, Nicaragua) } \\
\text { - Perception that clients would prefer FC } \\
\text { (Thailand) } \\
\text { - Bargaining tool for protected sex } \\
\text { (Bangladesh, Cambodia, Swaziland, Thailand) } \\
\text { - Share responsibility for condom use } \\
\text { (South Africa) } \\
\text { - Partner acceptance of first use predicted easier negotiation for } \\
\text { further uses } \\
\text { (Cote d'lvoire) } \\
\text { - Men would use if their partners initiate } \\
\text { (South Africa) } \\
\text { Potential for use during menstruation or breastfeeding protects } \\
\text { marriages } \\
\text { (Nigeria) } \\
\text { - Can be used if client/partner does not like/refuses/cannot use MC } \\
\text { (Brazil, India, South Africa, Bangladesh, Dominican Republic, } \\
\text { Thailand, El Salvador, Nicaragua, Nigeria) } \\
\text { - Better protection for women at risk of coerced sex } \\
\text { (Ghana) } \\
\text { - Better protection for women whose husband is unfaithful } \\
\text { (Vietnam) }\end{array}$ & $\begin{array}{l}\text { - Lack of perceived need (for barrier contraceptive) } \\
\text { (Kenya, Nigeria, Burundi, Bangladesh) } \\
\text { - Limited ability to discuss FC with partner } \\
\text { (Ghana) } \\
\text { - Desire for unprotected sex with a loving partner } \\
\text { (Nigeria, Uganda) } \\
\text { - Male partner resistance/refusal } \\
\text { (Ghana, Namibia, Nigeria, PNG, South Africa, Zimbabwe, } \\
\text { Burundi, El Salvador, Bangladesh, Cambodia, Swaziland, } \\
\text { Thailand, CAR) } \\
\text { - Fear of partner reaction/requirement for negotiation/ } \\
\text { partner cooperation } \\
\text { (Kenya, Uganda, Burundi, El Salvador) } \\
\text { - Male preference for being in control } \\
\text { (Zambia, Uganda) } \\
\text { - Men's fear that women reuse FC } \\
\text { (Uganda) } \\
\text { - Difficulty identifying strategies to negotiate FC use with } \\
\text { nonpaying partners } \\
\text { (El Salvador, Swaziland, Nicaragua) } \\
\text { - Clients accept MC } \\
\text { (Thailand) }\end{array}$ \\
\hline $\begin{array}{l}\text { Cultural and } \\
\text { religious norms }\end{array}$ & & $\begin{array}{l}\text { - Women unaccustomed, uncomfortable or embarrassed } \\
\text { to touch genitals/insert FC in front of a partner } \\
\text { (Brazil, South Africa, Cambodia, Dominican Republic) } \\
\text { - Embarrassment/anxiety over FC appearance and use } \\
\text { (Vietnam) } \\
\text { - Stigma/association with untrustworthiness/disease } \\
\text { (Ghana, Kenya, Nigeria, South Africa, Zimbabwe, } \\
\text { Burundi, El Salvador, Rwanda, Swaziland, Vietnam) } \\
\text { - Condom use conflicts with moral or religious beliefs } \\
\text { (Nigeria, Burundi, Bangladesh) }\end{array}$ \\
\hline $\begin{array}{l}\text { Promotion, } \\
\text { education, and } \\
\text { support }\end{array}$ & $\begin{array}{l}\text { - Provider or peer promotion/education/support/counseling } \\
\text { (Brazil, Kenya, Tanzania, People's Republic of China, South Africa) } \\
\text { - FC promoted as contraceptive rather than HIV prevention device } \\
\text { (Zimbabwe) }\end{array}$ & $\begin{array}{l}\text { - Lack of awareness of female anatomy causing fear of } \\
\text { losing FC in reproductive tract/abdomen } \\
\text { (Ghana, Zimbabwe, People's Republic of China, } \\
\text { South Africa) } \\
\text { - Lack of information about the device } \\
\text { (El Salvador, Nicaragua, Turkey, Tunisia) } \\
\text { - Not fully aware on how to use } \\
\text { (Malawi, South Africa) }\end{array}$ \\
\hline
\end{tabular}

Cultural and

religious norms

Promotion

education, an

FC promoted as contraceptive rather than HIV prevention device (Zimbabwe) 
Table 5 (Continued)

\begin{tabular}{|c|c|c|}
\hline Factor & $\begin{array}{l}\text { Facilitators } \\
\text { (countries where relevant evidence was identified) }\end{array}$ & $\begin{array}{l}\text { Barriers } \\
\text { (countries where relevant evidence was identified) }\end{array}$ \\
\hline Infrastructure & $\begin{array}{l}\text { - No need to attend clinic to access FCs } \\
\text { (Swaziland) }\end{array}$ & $\begin{array}{l}\text { - Difficulty disposing of FC } \\
\text { (India) } \\
\text { - Free FCs only at hospital } \\
\text { (Malawi) } \\
\text { - Difficulty of concealing the large package } \\
\text { (El Salvador, Nicaragua) }\end{array}$ \\
\hline $\begin{array}{l}\text { Poverty/financial } \\
\text { resources }\end{array}$ & $\begin{array}{l}\text { - For FSWs, allows higher earnings when used covertly with clients } \\
\text { requesting no condom use } \\
\text { (El Salvador, Nicaragua) } \\
\text { - For FSWs, allows higher earnings due to possibility of use during } \\
\text { menstruation } \\
\text { (El Salvador, Nicaragua) }\end{array}$ & $\begin{array}{l}\text { - Cost (if not free or heavily subsidized) } \\
\text { (Brazil, Ghana, India, Namibia, South Africa, Tanzania, } \\
\text { Zimbabwe, El Salvador, Mexico, Bangladesh, Nigeria, } \\
\text { Nicaragua, Malawi) } \\
\text { - Loss of potential earnings from clients requesting sex } \\
\text { without a condom } \\
\text { (El Salvador, Nicaragua) }\end{array}$ \\
\hline
\end{tabular}

Abbreviations: MC, male condom; FC, female condom; STI, sexually transmitted infection; PNG, Papua New Guinea; CAR, Central African Republic; FSW, female sex worker.

\section{Continued use}

A large number of studies reported that continued use was strongly influenced by experiences during the adoption phase. These included reservations regarding appearance, insertion or removal difficulties, discomfort caused by the inner or outer ring, noise, technical difficulties during sex, and partner resistance. ${ }^{60,66,71,92,96,102-106}$ However, study data indicated that most users overcome initial difficulties with practice, resulting in high acceptability ${ }^{62,70,81,102,107,108}$ and few clinical failures. ${ }^{63,90,95,107,109}$ For example, FSWs in El Salvador reported using FCs independently in up to ten sexual acts before they formed an opinion of the device and felt skilled enough to use it with a partner. Consequently, these women recommended that providers offer in-depth training to potential users who are new to the FCs. ${ }^{92}$

Continued use was commonly associated with adequate support during the adoption phase, through counseling or peer education, in studies following women and couples for 2-20 months. ${ }^{62,88,102,107,110}$ For example, in the People's Republic of China, education and demonstration sessions significantly increased FSWs' knowledge and acceptance of FCs, and confidence that clients would accept its use..$^{62,75}$ Brazilian women reported benefiting from FC demonstration, negotiation tips, hearing testimonials from others, and discussing initial difficulties:

The meetings were good because she explained many things, I could rehearse again how to insert it correctly in a model resembling the vagina, I also talked about how to introduce it to the partner. ${ }^{102}$

In the same Brazilian study, other important factors influencing continued use were perceived safety (compared with $\mathrm{MCs}$ ), pleasure (stimulation from external ring), and increased sense of power for safer sex negotiation. ${ }^{102}$

Conversely, some studies found that women were more likely to report inconsistent use or discontinuation when their initial difficulties were not overcome. Inconsistent use was most commonly attributed to partner objection - as cited by $30 \%$ of Kenyan women at 12 -month follow-up ${ }^{111}$ and by FSWs in Zimbabwe, who reported that their clients distrusted unfamiliar methods. ${ }^{63}$ Other common reasons for discontinuation were lack of perceived need for a barrier method, often due to belief in a mutually faithful partnership, ${ }^{111}$ or desire for unprotected sex with a loved one. ${ }^{48}$

Several studies found that discontinuation was frequently attributed to partner resistance, objection, or dislike of the device. ${ }^{60,66,112,113}$ Method attributes influencing discontinuation included unattractive appearance, noise, reduced sensation, size, overlubrication, difficulty inserting, and discomfort from the internal ring..$^{58,60,66,84,105,113,114}$

\section{Patterns of use}

National survey data (Table 3) supported a handful of crosssectional studies ${ }^{52,54,66,115}$ that demonstrated low ever or current use in the general population. FC use among FSWs was more common, ${ }^{64,67,85,116,117}$ with ever-use prevalence ranging from 5\% in Malawi ${ }^{104}$ to $33 \%$ in Swaziland. ${ }^{117}$

Notably, trends in uptake based on demographic variables were inconsistent between countries, and no strong association was apparent between awareness and ever use for 22 countries where both national datasets were available (Figure 1), although no formal statistical tests of associations were undertaken. An analysis of South African national survey data identified significant associations only with older 


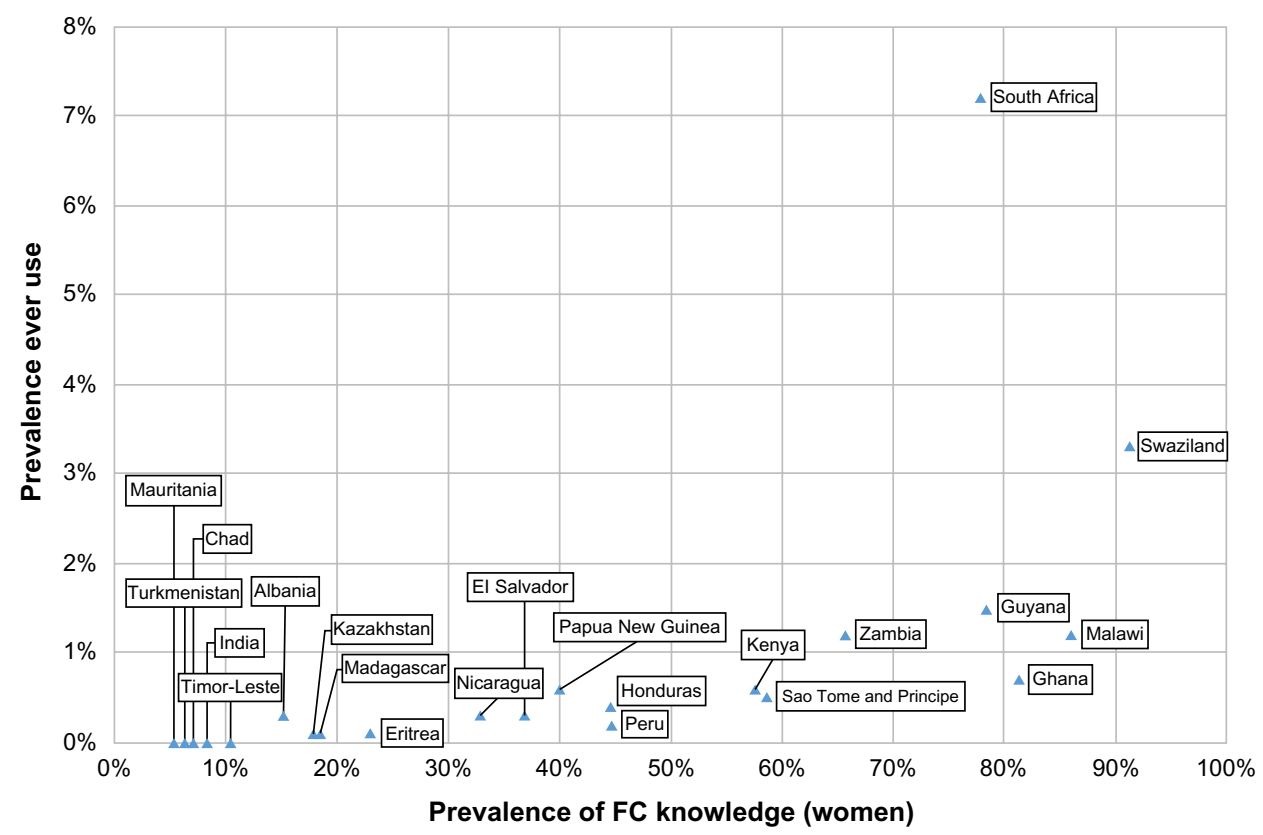

Figure I Scatterplot showing the prevalence of FC knowledge in women and ever use for countries where national survey data were available. Abbreviation: FC, female condom.

age and living in a particular province. Interestingly, many variables were associated with high knowledge prevalence but low use, or vice versa. ${ }^{55}$

Dual protection was a commonly cited advantage in a number of settings. For example, general population women in South Africa and Ghana usually cited STI/HIV prevention; ${ }^{66,88}$ Ugandan HIV-positive women cited prevention of partner transmission; ${ }^{118}$ and Swazi FSWs cited prevention of STIs, unintended pregnancy, and HIV reinfection. ${ }^{70}$ However, the most common partner type with whom FC use was reported varied between settings. For example, in Kenya, South Africa, and Zimbabwe, use was more common with a spouse/regular partner than a casual/commercial partner, ${ }^{61,69,88}$ whereas in Uganda, use was more common in high-risk sexual relationships. ${ }^{48}$ FSWs in Brazil and El Salvador reported using FCs more often with regular clients than with new/occasional clients or boyfriends. ${ }^{67,68}$ Conversely, despite both sexes generally perceiving dual protection as an advantage, women in Swaziland, Zimbabwe, Cote d'Ivoire, and India reported discomfort discussing disease prevention with long-term partners. ${ }^{69,70,119,120}$

\section{Consistency of use and impact on protected sex}

The prevalence of consistent FC use varied between studies, but it was often low. For instance, in Kenya, 11\% of women enrolled in an FC acceptability trial reported consistent use at 6-month follow-up. ${ }^{111}$ Conversely, consistent use in a general population sample ranged as high as $25 \%$, as shown in a recent cross-sectional study in a Zimbabwean hospital. ${ }^{121}$ Most evidence, however, suggested that couples interchange $\mathrm{MC}$ and $\mathrm{FC}$ use to maintain or increase the proportion of protected sex acts. ${ }^{69,70,88,122}$ In Zimbabwe, factors influencing method choice included availability, partner preference, and the woman's menstrual cycle. Women were more likely to use FCs consistently if they did not experience technical difficulties or partner opposition, did not rely on other contraceptive methods, and used FCs for contraception. ${ }^{69}$ Another Zimbabwean study showed that factors influencing consistent use depended on partner type: consistent use between spouses was negatively associated with multiple partner behaviors, but for regular nonmarital partners, it was positively associated with perceived ease of use and effectiveness for STI prevention. ${ }^{123}$

A 2006 systematic review ${ }^{77}$ and three further studies in Mexico, Kenya, and Madagascar ${ }^{85,122,124}$ concluded that FC provision can increase consistent condom use in a population, supporting other evidence that expanded choice improves contraceptive uptake and health outcomes. ${ }^{18,19}$ The addition of free $\mathrm{FC}$ provision into an existing peer education program among FSWs in Kenya increased consistent MC or FC use from $60 \%$ to $67 \%$. $^{124}$

\section{Covert use}

Despite being a commonly perceived advantage, studies that investigated actual covert FC use suggest that this practice is not widespread. Evidence from Brazil, South Africa, 
India, and Uganda suggested that only a minority of women practice covert use, ${ }^{43,60,67,125}$ although this may be higher for FSWs. ${ }^{126,127}$ In South Africa, reduced partner awareness, often when partners were drunk or high, was more important to women than absolute covert use. ${ }^{80}$ Covert use may facilitate higher sex work earnings from clients willing to pay more for sex without a condom, ${ }^{68}$ whilst increasing a woman's chance of protection:

[I]f you have the female condom, you can go to the bathroom and put it in and the client thinks they are not using a condom. But because you are wearing it, there is no risk that he can give you an infection (FSW, El Salvador) ${ }^{68}$

\section{Reuse}

Research in Zimbabwe, Uganda, Zambia, Mexico, Bangladesh, and Swaziland suggested that a minority of women reuse FCs. ${ }^{48,70,85,86,89,128}$ In India, 11\% of MSM users reported reuse of a single FC with multiple clients. ${ }^{42}$ In South Africa, among 150 family planning clients and women at high risk for STIs, 83\% reported willingness to reuse the FC, and those who trialed reuse up to seven times reported that the recommended steps involved were easy to perform and acceptable. ${ }^{91}$ In Swaziland, FSWs reported reuse without removal or washing, citing reasons of limited availability and lack of privacy needed for disposal and reinsertion. ${ }^{70}$

\section{Heterosexual male partners}

Studies involving heterosexual men were identified in 15 countries (Table 1); participants included single men, FSW clients, regular or cohabiting partners, and husbands. Men in several countries in Africa, Asia, and South America welcomed the device. ${ }^{45,59,87,102,115,129}$ For example, over $80 \%$ of men participating in an acceptability trial in India reported willingness to buy both the FC2 and Reddy FC in the future. ${ }^{45}$ Men frequently perceived superior effectiveness of the FC over the MC for HIV/STIs/pregnancy prevention and valued its dual protective properties. ${ }^{45,89,115,118,130}$ High levels of comfort and sensation were also reported, especially with practice, resulting from loose fit and lubrication. ${ }^{48,89,131}$ Men in Nigeria and Uganda liked the potential to insert before sex, and not having to remove the FC immediately after sex. ${ }^{48,89}$ Men in Brazil, South Africa, and China recognized the advantages of a female-initiated method, ${ }^{59,87,102,115}$ namely because it reduced male responsibility and increased female sexual agency: "Women can decide independently whether they would choose contraception or not. This is its greatest strength" (College educated young man, Shanghai). ${ }^{87}$
Conversely, several studies identified major concerns from men. ${ }^{48,59,79,82,130,132,133}$ Ugandan men complained that the FC was more difficult to use than the $\mathrm{MC},{ }^{48}$ while South African male students cited appearance, unfamiliarity, and concerns about pleasure as barriers to initial use, but felt that these might improve with experience. ${ }^{115}$ In another South African study, male students with prior use of the FC reported discomfort with their partners' suggestion of FC use, as well as female partners' insertion prior to negotiation. ${ }^{59}$ Other studies found that men felt threatened by a woman taking control of her own sexuality and were concerned that the FC might encourage promiscuity if women no longer feel at risk. ${ }^{82,130,132,133}$ Ugandan and Indian men feared that women would reuse $\mathrm{FC}$ without adequate cleaning. ${ }^{45,118}$

Negative attitudes were sometimes fuelled by misconceptions: men in Cameroon, Nigeria, and Zimbabwe reported a refusal to use FCs with FSWs for fear of multiple use or "sperm harvesting" for black magic. ${ }^{133}$ Some men in South Africa believed that they might not be protected by a device that is not worn by a man. ${ }^{115}$

Some studies found that women often reported positive responses from male partners, despite initial objection. ${ }^{63,88,102,134}$ For example, while some women in Zimbabwe reported partner refusal for fear of women becoming "casual about sex", most said they were encouraged to get more FCs. ${ }^{108}$ In South Africa, over $80 \%$ of women reported partner approval, based on natural feel, sexual pleasure, size, and strength. Conversely, attributes perceived as "disliked" were overlubrication and large size. ${ }^{50}$ Notably, these responses were mainly reported by women who had successfully negotiated FC use.

\section{Women's empowerment and negotiating use}

The relationship between FC and women's empowerment was frequently commented on, yet inconsistently interpreted by study authors. A few studies noted that by catalyzing processes that challenge established gender norms, FCs had the potential to transform gender relations. For example, university students in Nigeria reported that a key reason for FC use was the sense of empowerment that the method afforded. ${ }^{97}$ FC provision in Cambodia reinforced FSWs' intentions to share experiences and support each other to adopt new methods. ${ }^{127}$ In Mozambique, nearly 5,000 women have met in FC "empowerment groups" to discuss negotiation tactics with partners and the correct use of FCs, including basic education about women's bodies. ${ }^{135}$ Conversely, most authors 
characterized the $\mathrm{FC}$ as a protective tool for use within the existing constraints of gendered power imbalances, usually whereby women were empowered to protect themselves with the FC when their partner refused MC use. . $3,50,67,68,105,112,126,128^{-1}$ Commentaries were similarly mixed on the question of whether the FC truly "empowers", or whether it can only be used by already "empowered" women. ${ }^{12-14,125}$ In Tunisia, the authors of a study on FSWs commented that FC acceptance would be slow due to women's highly proscribed gender roles and the fact that "customs imposed a real obstacle to social innovations". ${ }^{103}$ A number of other studies found that FCs were of limited value to women, who were only able to use them with full partner awareness and agreement. ${ }^{82,125,136}$ Indeed, in one Zimbabwean study, two-thirds of women said that FC use depended on partner permission. ${ }^{82}$ Nonetheless, studies conducted in over half the countries found that the FC's female-initiated nature was perceived as a major advantage. In Zimbabwe, 35.6\% of female inconsistent condom users said they could use FCs more consistently than MCs, simply because they did not have to rely on the man to wear it. ${ }^{79,137}$ In Vietnam, women said that the FC offered more control over unwanted pregnancy, even if desired by their husbands, and it provided sexual security if they doubted their husbands' fidelity. ${ }^{81}$ Women in several settings preferred the FC to the MC because they could guarantee that it had not been deliberately damaged by men. ${ }^{70,126}$ FSWs often valued the potential for FC use with drunk clients, ${ }^{43,68,127,138}$ and some women saw benefit in inserting the FC before drinking themselves: "When I am going to date, and know I am going to drink, then I put the female condom before, because I think it is more safe, because I know that if I drink I can forget to put it on" (28-year-old drug user, Brazil). ${ }^{102}$

The $\mathrm{FC}$ is also used as a bargaining tool to negotiate either $\mathrm{MC}$ or FC use. ${ }^{127,128,138}$ For example, following an FC intervention in Thailand, $60 \%$ of FSWs reported that more clients agreed to use an $\mathrm{MC}$ after learning that the only alternative was the FC. ${ }^{138}$ Other gender dynamics reported include men's involvement in FC use; for example, one South African study found that $45 \%$ of women reported partner assistance with FC insertion, stabilizing the device, or removal. In this study, male involvement was most commonly reported by students and least commonly by FSWs. ${ }^{139}$

\section{Discussion and conclusion}

FCs can be highly acceptable to women and men with diverse risk profiles across a variety of settings, and as a female-initiated method, the device can be used as an additional tool to protect women within the context of gendered power imbalances. Furthermore, FCs are often used interchangeably with MCs, and thus their provision can positively impact the proportion of protected sex acts in a population, through uptake by women or couples for whom other methods of contraception or HIV prevention are inaccessible. The fact that most FC users employ the method interchangeably with the MC means that consistent $\mathrm{FC}$ use is less important than its role in increasing consistent condom use overall. Despite clear advantages at both an individual and population level, the national prevalence of FC use remains extremely low, even in the context of high awareness; these data suggest that models of successful programming are still not being implemented on a sufficiently wide scale. Current FC use was less than $1 \%$ (average: $0.04 \%$ ) for all countries, compared with current use of the IUD, pill, and injectables, which averaged between $2 \%-6 \%$, ranging up to $30 \%$ for the IUD (data not shown). ${ }^{33,35,36,41}$ Ever use of FC was also less than $1 \%$ for most countries - notable exceptions were South Africa (7.2\%), Swaziland (3.3\%), Guyana (1.5\%), Malawi (1.2\%), and Zambia (1.2\%) - compared with ever use of the IUD, pill, and injectables, which averaged between $9 \%-21 \%$, ranging up to nearly $50 \%$ for the IUD (data not shown) (Figure 1). ${ }^{33,36,41}$

Several important conclusions can be drawn from our data. Since the same method attributes are perceived positively and negatively within and between localities, contextual and environmental factors arguably play a greater role in determining overall FC acceptability and uptake. This is evidenced by the pervasive influence of stigma and male partner responses in determining initial and continued use of FCs. Indeed, even its female-initiated nature may limit acceptability if men fear loss of control. The fact that most of the physical and contextual factors negatively influencing use can be overcome with practice and adequate support suggests that demand creation is at least as important a component of programming as adequate supply.

Currently, since most research has focused on FC acceptability in trial settings, little is known about the profile of FC users and nonusers within the general population, and systems and market research to identify effective promotion and distribution mechanisms at a local level (ie, lower than national) is lacking. The scarcity of studies addressing FC use for anal sex by heterosexual couples and MSM in developing countries is an additional research gap, which perhaps reflects sociocultural taboo, as well as a general lack of attention to male perspectives on FC use. Policymakers, international donors, civil society groups, and programmers therefore 
have a responsibility not only to increase FC availability and advocate the sexual rights of women, but also to identify and implement local evidence-based strategies for effective FC programming.

There was near-universal endorsement, across the studies and population groups reviewed here, that increasing availability and access will contribute to increased FC use and limit reuse, although reuse levels were found to be low (current World Health Organization guidelines advise that a new FC should be used with every sexual act; Family Planning Global Handbook, 2011). ${ }^{141}$ Integrating FCs into a wide variety of services, programs, and nontraditional settings will inevitably improve access, but it may also increase exposure and normalize the device, thus contributing to the narrowing of the observed gap between awareness and use. Adequate programmatic support must also be available to ensure implementation of strategies with proven efficacy, such as FC demonstration, negotiation skills counseling, and user support. Greater efforts are also required to target men in FC programming and advocacy, by using male-specific branding and addressing potential anxieties related to female-initiated methods. One example is the recent branding as "inner condoms" by the South Africa-based Population Services International affiliate ${ }^{140}$ (see Figure 2). Promotional messaging should draw on local evidence to increase FC acceptability in the general population, by promoting commonly cited advantages such as sexual pleasure, while combating stigma and taboo.

\section{Limitations}

This review used peer-reviewed literature, country surveys, and other sources available via the Internet, and is thus subject to these limitations. Papers and reports outside our language scope would not be represented here. The fact that most research to date has been conducted with the discontinued FC1 suggests that some results may lack relevance to current programming. Our study was not intended to be a systematic review, and therefore did not include a complete count of articles retrieved and rejected. Scoping studies represent a broad approach to a topic, where the aim is to map a wide range of literature and identify the nature, range, and extent of the evidence. Some qualitative reports reviewed here were based on small sample sizes, although most were based on greater than 100 participants. Finally, the fact that our paper selected developing countries as a focus should not be construed to mean that the FC is not appropriate or acceptable for women in developed countries, as considerable literature has already shown.

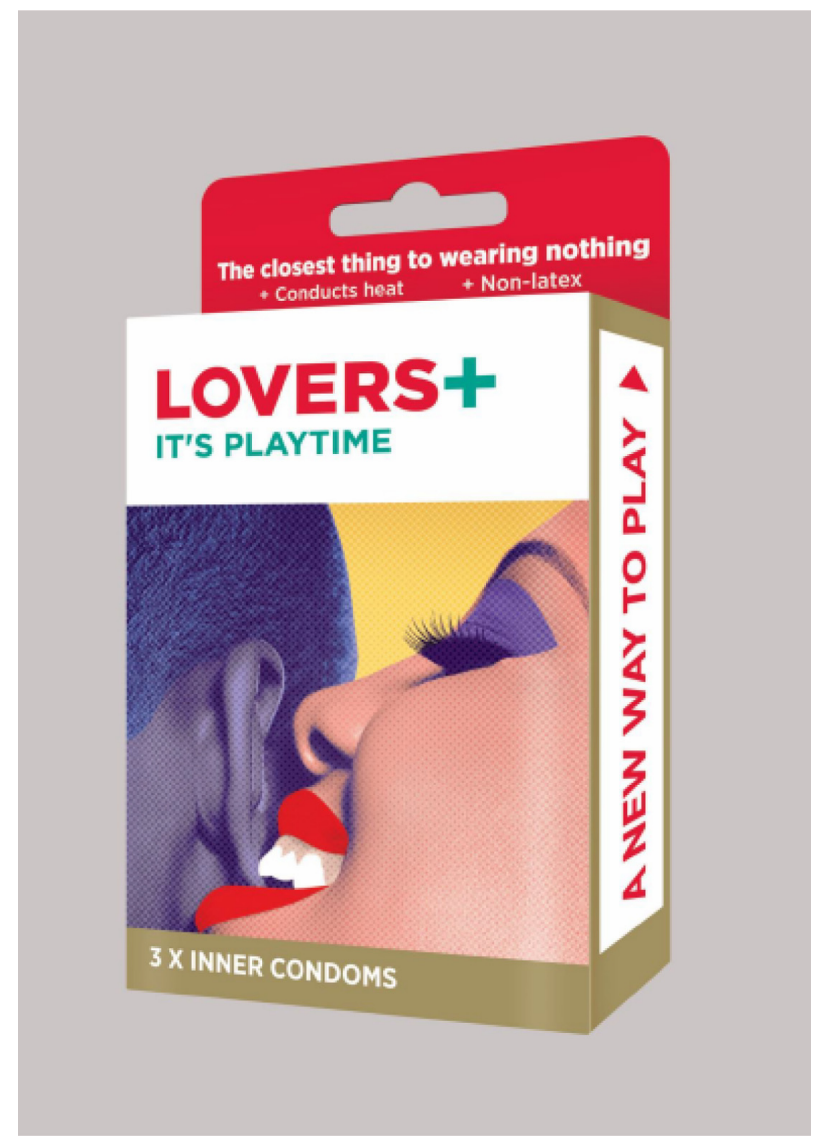

Figure 2 Lovers+ inner condoms.

\section{Disclosure}

The authors report no conflicts of interest in this work.

\section{References}

1. Joint United Nations Programme on HIV/AIDS (UNAIDS). UNAIDS Report on the Global AIDS Epidemic 2013. Geneva, Switzerland: Joint United Nations Programme on HIV/AIDS (UNAIDS); 2013.

2. Dunkle KL, Jewkes RK, Brown HC, Gray GE, McIntyre JA, Harlow SD. Gender-based violence, relationship power, and risk of HIV infection in women attending antenatal clinics in South Africa. Lancet. 2004; 363(9419):1415-1421.

3. Jewkes R, Morrell R. Gender and sexuality: emerging perspectives from the heterosexual epidemic in South Africa and implications for HIV risk and prevention. $J$ Int AIDS Soc. 2010;13:6.

4. Andersson N, Cockcroft A, Shea B. Gender-based violence and HIV: relevance for HIV prevention in hyperendemic countries of southern Africa. AIDS. 2008;22 Suppl 4:S73-S86.

5. Dunkle KL, Stephenson R, Karita E, et al. New heterosexually transmitted HIV infections in married or cohabiting couples in urban Zambia and Rwanda: an analysis of survey and clinical data. Lancet. 2008; 371(9631):2183-2191.

6. Hirsch JS, Meneses S, Thompson B, Negroni M, Pelcastre B, del Rio C. The inevitability of infidelity: sexual reputation, social geographies, and marital HIV risk in rural Mexico. Am J Public Health. 2007;97(6): 986-996.

7. Clark. Early marriage and HIV risks in sub-Saharan Africa. Study Fam Plann. 2004;35(3):149-160. 
8. Sedgh G, Hussain R, Bankole A, Singh S. Women with an Unmet Need for Contraception in Developing Countries and Their Reasons for Not Using a Method, Occasional Report No 37. New York, NY: Guttmacher Institute; 2007.

9. Alkema L, Kantorova V, Menozzi C, Biddlecom A. National, regional, and global rates and trends in contraceptive prevalence and unmet need for family planning between 1990 and 2015: a systematic and comprehensive analysis. Lancet. 2013;381(9878): 1642-1652.

10. Gollub E, Stein Z. Living with uncertainty: acting in the best interests of women. AIDS Res Treat. 2012;2012:524936.

11. Gallo MF, Kilbourne-Brook M, Coffey PS. A review of the effectiveness and acceptability of the female condom for dual protection. Sex Health. 2012;9(1):18-26.

12. Gollub EL. The female condom: tool for women's empowerment. Am J Public Health. 2000;90(9):1377-1381.

13. Mantell JE, Stein ZA, Susser I. Women in the time of AIDS: barriers, bargains, and benefits. AIDS Educ Prev. 2008;20(2):91-106.

14. PATH; UNFPA. Female Condo: A Powerful Tool for Protection. Seattle, WA: UNFPA and PATH; 2006

15. Beksinska M, Smit J, Joanis C, Potter W. New female condoms in the pipeline. Reprod Health Matters. 2012;20(40):188-196.

16. United Nations Population Fund. UNFPA Prequalified Female Condom Manufacturing Sites. New York, NY: United Nations Population Fund; 2013.

17. Peters A, Jansen W, van Driel F. The female condom: the international denial of a strong potential. Reprod Health Matters. 2010;18(35): 119-128.

18. Warren M, Philpott A. Expanding safer sex options: introducing the female condom into national programmes. Reprod Health Matters. 2003;11(21):130-139.

19. Marseille E, Kahn JG. Smarter Programming of the Female Condom: Increasing Its Impact on HIV Prevention in the Developing World. San Francisco, CA: FSG Social Impact Advisors; 2008.

20. United Nations Population Fund. Donor Support for Contraceptives and Condoms for Family Planning and STI/HIV Prevention 2010. New York, NY: UNFPA; 2010.

21. United States Agency for International Development. Is There a Condom Gap In 2010? Washington, DC: USAID; 2010.

22. Center for Health and Gender Equity. Saving Lives Now: Female Condoms and the Role of US Foreign Aid. Takoma Park, MD: Center for Health and Gender Equity; 2008.

23. Hoffman S, Mantell J, Exner T, Stein Z. The future of the female condom. Int Fam Plan Perspect. 2004;30(3):139-145.

24. Dowdy DW, Sweat MD, Holtgrave DR. Country-wide distribution of the nitrile female condom (FC2) in Brazil and South Africa: a costeffectiveness analysis. AIDS. 2006;20(16):2091-2098.

25. Marseille E, Kahn JG, Billinghurst K, Saba J. Cost-effectiveness of the female condom in preventing HIV and STDs in commercial sex workers in rural South Africa. Soc Sci Med. 2001;52(1):135-148.

26. World Health Organization. Making Decisions about Contraceptive Introduction: A Guide for Conducting Assessments to Broaden Contraceptive Choice and Improve Quality of Care. Geneva, Switzerland: World Health Organization; 2002.

27. Minnis AM, Shiboski SC, Padian NS. Barrier contraceptive method acceptability and choice are not reliable indicators of use. Sex Transm Dis. 2003;30(7):556-561.

28. Mantell JE, Scheepers E, Karim QA. Introducing the female condom through the public health sector: experiences from South Africa. AIDS Care. 2000;12(5):589-601.

29. Levac D, Colquhoun H, O’Brien KK. Scoping studies: advancing the methodology. Implement Sci. 2010;5:69.

30. Arskey H, O’Malley L. Scoping studies: towards a methodological framework. Int J Soc Res Methodol. 2005;8(1):19-32.

31. Reproductive Health Supplies Coalition. Supplies Information Database. Brussels, Belgium: Reproductive Health Supplies Coalition; 2014.
32. The Female Health Company. Female Condom Bibliography - Sorted by Date. Chicago, IL: The Female Health Company; 2013. Available from: http://www.femalecondom.org/wp-content/uploads/2013/07/ Female_condom_bibliography_march_2013_date.pdf. Accessed May 11, 2015.

33. ICF International. The DHS Program STATcompiler. Rockville, MD: ICF International; 2012.

34. Ministry of Health and Social Services. Namibia Demographic and Health Survey. Windhoek, Namibia: Ministry of Health and Social Services; 2014.

35. Central Statistical Office; UNICEF. Swaziland Multiple Indicator Cluster Survey 2010. Mbabane, Swaziland: Central Statistical Office and UNICEF; 2011.

36. Shisana O, Rehle T, Simbayi LC, et al. South African National HIV Prevalence, Incidence, Behaviour and Communication Survey 2008: A Turning Tide among Teenagers? Cape Town, South Africa: HSRC Press; 2008.

37. National Department of Health. South Africa Demographic and Health Survey 2003. Pretoria, South Africa: National Department of Health; 2003.

38. Centro de Estudios Sociales Y Demograficos. República Dominicana Encuesta Demografica Y de Salud 2007. Calverton, MD: Macro International Inc.; 2008. Spanish.

39. National Statistical Office. Papua New Guinea Demographic and Health Survey 2006. Port Moresby, Papua New Guinea: National Statistical Office; 2009.

40. National Population Commission. Nigeria Demographic and Health Survey 2008. Calverton, MD: ICF Macro; 2008.

41. Ministry of Health and Family Welfare. India National Family Health Survey (NFHS-3) 2005-6. Mumbai, India: International Institute for Population Sciences; 2007.

42. Kokku SB, Tucker S, Punyam S. Exploring dynamics around female condom use for anal sex among MSM. Sex Transm Infect. 2011;87:A250.

43. Hindustan Latex Family Planning Promotion Trust. Female Condom: The Indian Experience. London, UK: The Female Health Company; 2004.

44. Chen B, Zhang M, Yang D, Wang X, Zheng Y. Acceptability of female condom use in money boys compared with other men who have sex with men in Chengdu, China: a comparative study. Sex Health. 2011;8(2):262-263

45. Smita J, Neelam J, Rochelle DY, et al. Comparative acceptability study of the Reality female condom and the version 4 of modified Reddy female condom in India. Contraception. 2005;72(5): 366-371.

46. Joanis C, Beksinska M, Hart C, Tweedy K, Linda J, Smit J. Three new female condoms: which do South-African women prefer? Contraception. 2011;83(3):248-254.

47. Beksinska ME, Piaggio G, Smit JA, et al. Performance and safety of the second-generation female condom (FC2) versus the Woman's, the VA worn-of-women, and the Cupid female condoms: a randomised controlled non-inferiority crossover trial. Lancet Glob Health. 2013;1(3): e146-e152.

48. Wanyenze RK, Atuyambe L, Kibirige V, et al. The new female condom (FC2) in Uganda: perceptions and experiences of users and their sexual partners. Afr J AIDS Res. 2011;10(3):219-224.

49. Hou LY, Qiu HY, Zhao YZ, Zeng XS, Cheng YM. A crossover comparison of two types of female condom. Int J Gynaecol Obstet. 2010; 108(3):214-218.

50. Smit J, Beksinska M, Vijayakumar G, Mabude Z. Short-term acceptability of the reality polyurethane female condom and a synthetic latex prototype: a randomized crossover trial among South African women. Contraception. 2006;73(4):394-398.

51. Ministère du Plan et Suivi de la Mise en œuvre de la Révolution de la Modernité/Ministère de la Santé Publique. Enquête Démographique et de Santé En République Démocratique Du Congo 2013-2014. Rockville, MD: ICF International; 2014. French. 
52. Oladeinde BH, Omoregie R, Onifade AA, Olley M, Anunibe JA, Oladeinde OB. Awareness and use of female condoms among young Nigerian women. Centrepoint Journal. 2011;17(2):157-163.

53. Çalışkan D, Sakızlıgil BH, Ocaktan ME. The awareness and use of the female condom among women at low and high risk for sexually transmitted infections in Ankara, Turkey. Dicle Medical Journal/Dicle Tip Dergisi. 2012;39(1):9.

54. Mbarushimana V, Ntaganira J. Knowledge and attitude to female condom use among undergraduates of Kigali Health Institute. Rwanda Journal of Health Sciences. 2013;2(1):16-25.

55. Guerra FM, Simbayi LC. Prevalence of knowledge and use of the female condom in South Africa. AIDS Behav. 2014;18(1):146-158.

56. Central Statistical Office. Swaziland Demographic and Health Survey. Calverton, MD: Macro International Inc.; 2008

57. Xu JX, Leeper MA, Wu Y, et al. User acceptability of a female condom (Reality) in Shanghai. Adv Contracept. 1998;14(4):193-199.

58. Barbosa RM, Kalckmann S, Berquó E, Stein Z. Notes on the female condom: experiences in Brazil. Int J STD AIDS. 2007;18(4):261-266.

59. Masvawure TB, Mantell JE, Mabude Z, et al. "It's a different condom, let's see how it works": young men's reactions to and experiences of female condom use during an intervention trial in South Africa. $J$ Sex Res. 2014;51(8):841-851.

60. Beksinska ME, Rees VH, McIntyre JA, Wilkinson D. Acceptability of the female condom in different groups of women in South Africaa multicentred study to inform the national female condom introductory strategy. S Afr Med J. 2001;91(8):672-678.

61. Liverpool VCT, Care, and Treatment, Population Council. FemaleInitiated Prevention: Integrating Female Condoms into HIV Risk-Reduction Activities in Kenya. Nairobi, Kenya: Population Council; 2009.

62. Yimin C, Zhaohui L, Xianmi W, et al. Introductory study on female condom use among sex workers in China. Contraception. 2002;66(3):179-185.

63. Ray S, van De Wijgert J, Mason P, Ndowa F, Maposhere C. Constraints faced by sex workers in use of female and male condoms for safer sex in urban Zimbabwe. J Urban Health. 2001;78(4):581-592.

64. United States Agency for International Development/Family Health International. Behaviors, Knowledge, Exposure to Interventions. Port Moresby, Papua New Guinea: FHI Papua New Guinea; 2011.

65. Adeokun L, Mantell JE, Weiss E, et al. Promoting dual protection in family planning clinics in Ibadan Nigeria. Int Fam Plan Perspect. 2002;28(2):87-95.

66. Skaer M, Ebin V. In Our Own Hands: SWAA-Ghana Champions the Female Condom. New York, NY: Population Council; 2006.

67. Galvão LW, Diaz J, Mobley S, Cabral F, Marchi N, Fruet S. Acceptability of the Female Condom After a Social Marketing Campaign in Campinas, Brazil. Washington, DC: Population Council; 2002.

68. Mack N, Grey TG, Amsterdam A, Matta CI, Williamson N. Central American sex workers' introduction of the female condom to different types of sexual partners. AIDS Educ Prev. 2010;22(5):466-481.

69. Kerrigan D, Mobley S, Rutenberg N, Fisher A, Weiss E. The Female Condom: Dynamics of Use in Urban Zimbabwe. Washington, DC: Population Council; 2000.

70. Mathenjwa T, Maharaj P. 'Female condoms give women greater control': a qualitative assessment of the experiences of commercial sex workers in Swaziland. Eur J Contracept Reprod Health Care. 2012; 17(5):383-392.

71. Ezire O, Oluigbo O, Archibong V, Ifeanyi O, Anyanti J. Barriers to repeated use of female condom among women and men of reproductive age in Nigeria. Journal of AIDS and HIV Research. 2013;5(6): 206-213.

72. Rasch V, Yambesi F, Kipingili R. Acceptance and use of the female condom among women with incomplete abortion in rural Tanzania. Contraception. 2007;75(1):66-70.

73. Ara Z, Begum N, Nweneka CV. Acceptability of female-controlled HIV/STI prevention options among professionals in Dhaka, Bangladesh. South East Asia Journal of Public Health. 2012;2(1):46-53.

74. Agha S. Intention to use the female condom following a mass-marketing campaign in Lusaka, Zambia. Am J Public Health. 2001;91(2): 307-310.
75. Liao S, Weeks MR, Wang $\mathrm{Y}$, et al. Inclusion of the female condom in a male condom-only intervention in the sex industry in China: a crosssectional analysis of pre- and post-intervention surveys in three study sites. Public Health. 2011;125(5):283-292.

76. Wang Y, Liao S, Jiang J, et al. Who are the preferential targets for intervention programs related to the female condom among sex workers in southern China? AIDS Educ Prev. 2013;25(4):349-361.

77. Vijayakumar G, Mabude Z, Smit J, Beksinska M, Lurie M. A review of female-condom effectiveness: patterns of use and impact on protected sex acts and STI incidence. Int J STD AIDS. 2006;17(10): 652-659.

78. Munyana M. Promoting the female condom in Burundi. Exchange on HIV/AIDS, Sexuality and Gender. 2006;2:12-13.

79. Buck J, Kang MS, van der Straten A, Khumalo-Sakutukwa G, Posner S, Padian N. Barrier method preferences and perceptions among Zimbabwean women and their partners. AIDS Behav. 2005;9(4):415-422.

80. MacPhail C, Terris-Prestholt F, Kumaranayake L, Ngoako P, Watts C, Rees H. Managing men: women's dilemmas about overt and covert use of barrier methods for HIV prevention. Cult Health Sex. 2009;11(5): 485-497.

81. United Nations Population Fund. The Acceptability of Female Condoms among Migrant Workers in Industrial Zones in Viet Nam. Ha Noi, Viet Nam: United Nations Population Fund; 2012.

82. Francis-Chizororo M, Natshalaga NR. The female condom: acceptability and perception among rural women in Zimbabwe. Afr J Reprod Health. 2003;7(3):101-116.

83. Susser I, Stein Z. Culture, sexuality, and women's agency in the prevention of HIV/AIDS in southern Africa. Am J Public Health. 2000;90(7): 1042-1048.

84. UNAIDS; STI/HIV/AIDS Prevention Center; World Health Organization. Needs and Acceptability of Female Condoms among Women in Thanh Xuan Commune and Dong Da District, Hanoi. Hanoi, Vietnam: UNAIDS, STI/HIV/AIDS Prevention Center, and WHO; 2000.

85. Stockman JK, Morris MD, Martinez G, et al. Prevalence and correlates of female condom use and interest among injection drug-using female sex workers in two Mexico-US border cities. AIDS Behav. 2012;16(7):1877-1886.

86. Smith JB, Nkhama G, Trottier DA. Female condom reuse in Lusaka, Zambia: evidence from 12 cases. J Urban Health. 2001;78(4): 638-646.

87. Coffey PS, Kilbourne-Brook M, Junqing W, et al. Initial reactions to the Woman's Condom by potential user groups in Shanghai, China. J Fam Plann Reprod Health Care. 2013;39(2):111-120.

88. Reproductive Health Research Unit, Family Health International and the Department of Health of South Africa. Introduction of the female condom in South Africa: programme activities and performance 1998-2000. Draft report in preparation.

89. National AIDS Coordination Programme. Piloting the Female Condom in Zimbabwe: A National Survey. Harare, Zimbabwe: Ministry of Health and Child Welfare; 1998.

90. Zachariah R, Harries AD, Buhendwa L, Spielman MP, Chantulo A, Bakali E. Acceptability and technical problems of the female condom amongst commercial sex workers in a rural district of Malawi. Trop Doct. 2003;33(4):220-224

91. Pettifor AE, Beksinska ME, Rees HV, Mqoqi N, Dickson-Tetteh KE. The acceptability of reuse of the female condom among urban South African women. J Urban Health. 2001;78(4):647-657.

92. Mack N, Grey TG, Amsterdam A, Williamson N, Matta CI. Introducing female condoms to female sex workers in Central America. Int Perspect Sex Reprod Health. 2010;36(3):149-155.

93. Tarkang EE, Bain LE. Factors that influence utilization of the female condom among senior secondary school female students in urban Cameroon. American Journal of Health Research. 2014;2(4): $125-133$.

94. Holt K, Blanchard K, Chipato T, et al. A nationally representative survey of healthcare provider counselling and provision of the female condom in South Africa and Zimbabwe. BMJ Open. 2013;3(3). pii: e002208. 
95. Wang X, Xi M, Zhang L, Jia L, Wang Y, Cheng Y. Awareness of female condoms and failures reported with two different types in China. Int J Gynaecol Obstet. 2015;128(2):152-156.

96. Séhonou J, Grésenguet G, Tokunaga M, Mbopi Kéou FX, Favier C, Bélec L. Acceptability of the female condom in Bangui, Central African Republic: results from a prospective study. Int J STD AIDS. 2002;13(9):649-651.

97. Tobin-West CI, Maduka O, Onyekwere VN, Tella AO. Awareness, acceptability, and use of female condoms among university students in Nigeria: implications for STI/HIV prevention. Int J Adolesc Med Health. 2014;26(2):259-265.

98. Ankrah EM, Attika SA. Adopting the Female Condom in Kenya and Brazil: Perspectives of Women and Men. A Synthesis. Arlington, VA: Family Health International (FHI) and AIDS Control and Prevention Project (AIDSCAP); 1997.

99. Agha S, Van Rossem R. Impact of mass media campaigns on the intentions to use the female condom in Tanzania. Int Fam Plan Perspect. 2002;28(3):151-158.

100. Mantell JE, Smit JA, Exner TM, et al. Promoting female condom use among female university students in KwaZulu-Natal, South Africa: results of a randomized behavioral trial. AIDS Behav. Epub August 5, 2014.

101. Mung'ala L, Kilonzo N, Angala P, Theobald S, Taegtmeyer M. Promoting female condoms in HIV voluntary counselling and testing centres in Kenya. Reprod Health Matters. 2006;14(28):99-103.

102. Telles Dias PR, Souto K, Page-Shafer K. Long-term female condom use among vulnerable populations in Brazil. AIDS Behav. 2006; 10(4 Suppl):S67-S75.

103. Boudaya S, Masmoudi A, Meziou TJ, et al. [Acceptability of the female condom among prostitutes in Tunisia]. Ann Dermatol Venereol. 2006;133(2):185-187. French.

104. Family Planning Association in Malawi. Counting the Uncatchables: A Report of the Situation Analysis of the M. Lagnitude, Behavioural Patterns, Contributing Factors, current Interventions and Impact of Sex Work in HIV Prevention in Malawi. Lilongwe, Malawi: Family Planning Association in Malawi and UNFPA; 2011.

105. Naidu M. Perceptions around second generation female condoms reporting on women's experiences. Anthropological Notebooks. 2013;19(1):25-34.

106. Okunlola MA, Morhason-Bello IO, Owonikoko KM, Adekunle AO. Female condom awareness, use and concerns among Nigerian female undergraduates. J Obstet Gynaecol. 2006;26(4):353-356.

107. Galvão LW, Oliveira LC, Díaz J, et al. Effectiveness of female and male condoms in preventing exposure to semen during vaginal intercourse: a randomized trial. Contraception. 2005;71(2):130-136.

108. Ray S, Bassett M, Maposhere C, et al. Acceptability of the female condom in Zimbabwe: positive but male-centred responses. Reprod Health Matters. 1995;3(5):68-79.

109. Beksinska M, Smit J, Greener R, Piaggio G, Joanis C. The female condom learning curve: patterns of female condom failure over 20 uses. Contraception. 2015;91(1):85-90.

110. Hoke TH, Feldblum PJ, Damme KV, et al. Randomised controlled trial of alternative male and female condom promotion strategies targeting sex workers in Madagascar. Sex Transm Infect. 2007;83(6):448-453.

111. Feldblum PJ, Kuyoh MA, Bwayo JJ, et al. Female condom introduction and sexually transmitted infection prevalence: results of a community intervention trial in Kenya. AIDS. 2001;15(8):1037-1044.

112. Sakondhavat $C$, Weeravatrakul $Y$, Benette $T$, et al. Consumer preference study of the female condom in a sexually active population at risk of contracting AIDS. J Med Assoc Thai. 2001;84(7):973-981.

113. Isah $Y$, Ogunbowale D, Alagbile M. Acceptability of female condom as a family planning method among women of reproductive ages in Adamawa State, North Eastern Nigeria. International Conference on Family Planning; November 12-15, 2013; Addis Ababa, Ethiopia.

114. Sinpisut P, Chandeying V, Skov S, Uahgowitchai C. Perceptions and acceptability of the female condom [Femidom] amongst commercial sex workers in the Songkla province, Thailand. Int J STD AIDS. 1998;9(3):168-172.
115. Mantell JE, Smit JA, Beksinska M, et al. Everywhere you go, everyone is saying condom, condom. But are they being used consistently? Reflections of South African male students about male and female condom use. Health Educ Res. 2011;26(5):859-871.

116. United States Agency for International Development/Namibia. HIV and AIDS and Treatment Literacy Namibia Survey 2008: Main Report. Windhoek, Namibia: USAID/Namibia; 2009.

117. National Emergency Response Council on HIV and AIDS and United Nations Programme on HIV/AIDS. Situation Analysis on Commercial Sex Work in Swaziland. Mbabane, Swaziland: NERCHA and UNAIDS; 2007.

118. Wanyenze RK, Tumwesigye NM, Kindyomunda R, et al. Uptake of family planning methods and unplanned pregnancies among HIVinfected individuals: a cross-sectional survey among clients at HIV clinics in Uganda. $J$ Int AIDS Soc. 2011;14:35.

119. Usmani F. The Female Condom: Opportunities and Challenges in South Asia. Kathmandu, Nepal: UNFPA Country Technical Services Team for South and West Asia; 2002.

120. Deniaud F. [Dynamics of female condom acceptability among prostitutes and young women in Abidjan, Ivory Coast]. Contracept Fertil Sex. 1997;25(12):921-932. French.

121. Chipfuwa T, Manwere A, Kuchenga MM, et al. Level of awareness and uptake of the female condom in women aged 18 to 49 years in Bindura district, Mashonaland Central province, Zimbabwe. Afr J AIDS Res. 2014;13(1):75-80.

122. Hoke TH, Feldblum PJ, Van Damme K, et al. Temporal trends in sexually transmitted infection prevalence and condom use following introduction of the female condom to Madagascar sex workers. Int $J$ STD AIDS. 2007;18(7):461-466.

123. Meekers D, Richter K. Factors associated with use of the female condom in Zimbabwe. Int Fam Plan Perspect. 2005;31(1):30-37.

124. Thomsen SC, Ombidi W, Toroitich-Ruto C, et al. A prospective study assessing the effects of introducing the female condom in a sex worker population in Mombasa, Kenya. Sex Transm Infect. 2006;82(5): 397-402.

125. Green G, Pool R, Harrison S, et al. Female control of sexuality: illusion or reality? Use of vaginal products in south west Uganda. Soc Sci Med. 2001;52(4):585-598.

126. van Dijk MG, Pineda DL, Grossman D, Sorhaindo A, García SG. The female condom: a promising but unavailable method for Dominican sex workers, their clients, and their partners. J Assoc Nurses AIDS Care. 2013;24(6):521-529.

127. Busza J, Baker S. Protection and participation: an interactive programme introducing the female condom to migrant sex workers in Cambodia. AIDS Care. 2004;16(4):507-518.

128. Sharifuzzaman M, Jana S, Gomes JM, Enamul Hoque ASM, Mahmud KF. Female condom (FC) can lead safer sex when clients do not agree to use condom. In: The XIV International AIDS Conference; 2002: Abstract MoPeF4025.

129. Blogg J, Blogg S. Acceptability of the Female Condom (Femidom) Within a Population of Commercial Sex Workers and Couples in Salima Nkhotakota, Malawi. London, UK: The Female Health Company; 1994.

130. Pool R, Hart G, Green G, Harrison S, Nyanzi S, Whitworth J. Men's attitudes to condoms and female controlled means to protect against HIV and STDs in south-western Uganda. Cult Health Sex. 2000;2(2):197-211.

131. Coffey PS, Kilbourne-Brook M, Austin G, Seamans Y, Cohen J. Shortterm acceptability of the PATH Woman's Condom among couples at three sites. Contraception. 2006;73(6):588-593.

132. Mantell JE, Adeokun L, Weiss E, et al. The impact of male gender roles on HIV risk in southwest Nigeria. In: The 129th Annual Meeting of APHA. Atlanta, GA: American Public Health Association; 2001.

133. Koster W, Bruinderink MG, Kuijper C, Siemerink MC. Male views on acceptability and use of female condoms with sex workers: findings from a qualitative study in Nigeria, Cameroon and Zimbabwe. In: The 19th International AIDS Conference. Washington, DC: International AIDS Society; 2012: Abstract no THPE203. 
134. Susser I. AIDS, Sex and Culture: Global Politics and Survival in Southern Africa. Chichester, UK: Wiley-Blackwell; 2009.

135. Simone O, Vespia $M$ [webpage on the Internet]. The power of female condoms in Mozambique. Watertown, MA: Pathfinder International; 2013. Available from: http://www.pathfinder.org/blog/to-youngwomen-in-mozambique.html. Accessed.

136. Hart GJ, Pool R, Green G, Harrison S, Nyanzi S, Whitworth JA. Women's attitudes to condoms and female-controlled means of protection against HIV and STDs in south-western Uganda. AIDS Care. 1999;11(6):687-698.

137. Napierala S, Kang MS, Chipato T, Padian N, van der Straten A. Female condom uptake and acceptability in Zimbabwe. AIDS Educ Prev. 2008;20(2):121-134.

138. Jivasak-Apimas S, Saba J, Chandeying V, et al. Acceptability of the female condom among sex workers in Thailand: results from a prospective study. Sex Transm Dis. 2001;28(11):648-654.
139. Beksinska ME, Rees HV, Kleinschmidt I, McIntyre J. The practice and prevalence of dry sex among men and women in South Africa: a risk factor for sexually transmitted infections? Sex Transm Infect. 1999;75(3):178-180.

140. Population Services International (PSI) South Africa. Lovers+ It's Playtime. PSI.

141. World Health Organization Department of Reproductive Health and Research (WHO/RHR); Johns Hopkins Bloomberg School of Public Health/Center for Communication Programs (CCP) Knowledge for Health Project. Family Planning: A Global Handbook for Providers (2011 Update). Baltimore, MD and Geneva, Switzerland: CCP and WHO; 2011.
Open Access Journal of Contraception

\section{Publish your work in this journal}

Open Access Journal of Contraception is an international, peerreviewed, open access, online journal, publishing original research, reports, reviews and commentaries on all areas of contraception. In addition to clinical research, demographics and health-related aspects, the journal welcomes new findings in animal and preclinical studies

\section{Dovepress}

relating to understanding the biological mechanisms and practical development of new contraceptive agents. The manuscript management system is completely online and includes a very quick and fair peer-review system. Visit http://www.dovepress.com/testimonials.php to read real quotes from published authors. 